\title{
Observing Nebulosities: The Cygnus Superbubble
}

\author{
Chris Christopherson ${ }^{1}$, Nadia Kaltcheva \\ Department of Physics and Astronomy \\ University of Wisconsin Oshkosh \\ Oshkosh, WI 54901
}

\begin{abstract}
The Galactic star-forming complexes are best delineated by the distribution of the ionized interstellar matter which is always associated with active star-formation. Observations of these regions at specific emission lines are helpful in determining the overall distribution of the interstellar gas and dust, and also tracing supernova shock fronts and regions of strong stellar winds. Amateur astrophotography equipment is often useful in obtaining homogeneous coverage of large-scale starforming fields that in some cases does not yet exist as part of professional all-sky surveys. For this project we observe the Cygnus star-forming complex in the Hydrogen- $\alpha$, Hydrogen- $\beta$ and Oxygen-III $(500.7 \mathrm{~nm})$ emission lines. We use a computer-guided modified Digital Single Lens Reflex (DSLR) camera combined with a wide-angle lens to image a $22^{\circ} \times 17^{\circ}$ field, including in this way all prominent star-formation sites in Cygnus.
\end{abstract}

\section{Introduction}

The Cygnus star-forming complex is one of the largest isolated features of interstellar gas and dust recognized in the Milky Way. In the sky, it is associated with the Northern Cross asterism, the bright supergiant star Deneb and the picturesque North America and Pelican emission nebulae (Fig. 1). Observations in the X-ray, radio and optical part of the spectrum reveal a giant ring of hot gas of about $400 \mathrm{pc}$ in diameter, known as the Cygnus superbubble. Filled with regions of star formation, it is surrounded by a shell of gaseous filaments and obscuring dust clouds known as the Great Cygnus Rift. The structure and the energetics of this extended region of expanding interstellar matter are still not well understood. Since, within the Milky Way, Cygnus is located along the local Orion spur, star-forming structures at different distances are possibly located along the line of sight. Indeed, recent X-ray emission findings (Uyaniker et al. 2001) suggest that the dominant nebulosity in the Cygnus field, Sh 2-109 (Sharpless, 1959), consists of overlapping, not physically related, features.

According to current theories, stars form in clouds of interstellar gas and dust. Only a small fraction of the newly born stars are significantly greater in mass than the Sun, but they emit vast amounts of high-energy radiation into the surrounding space. Their radiation pressure, in turn, triggers the formation of more stars. Studying the interplay between stars and interstellar matter allow us to reveal key aspects of the star-formation process.

\footnotetext{
${ }^{1}$ Research done with the support of the Wisconsin Space Grant Consortium, NASA Space Grant College and Fellowship Program, NASA Training Grant \#NNX14AP22H
} 


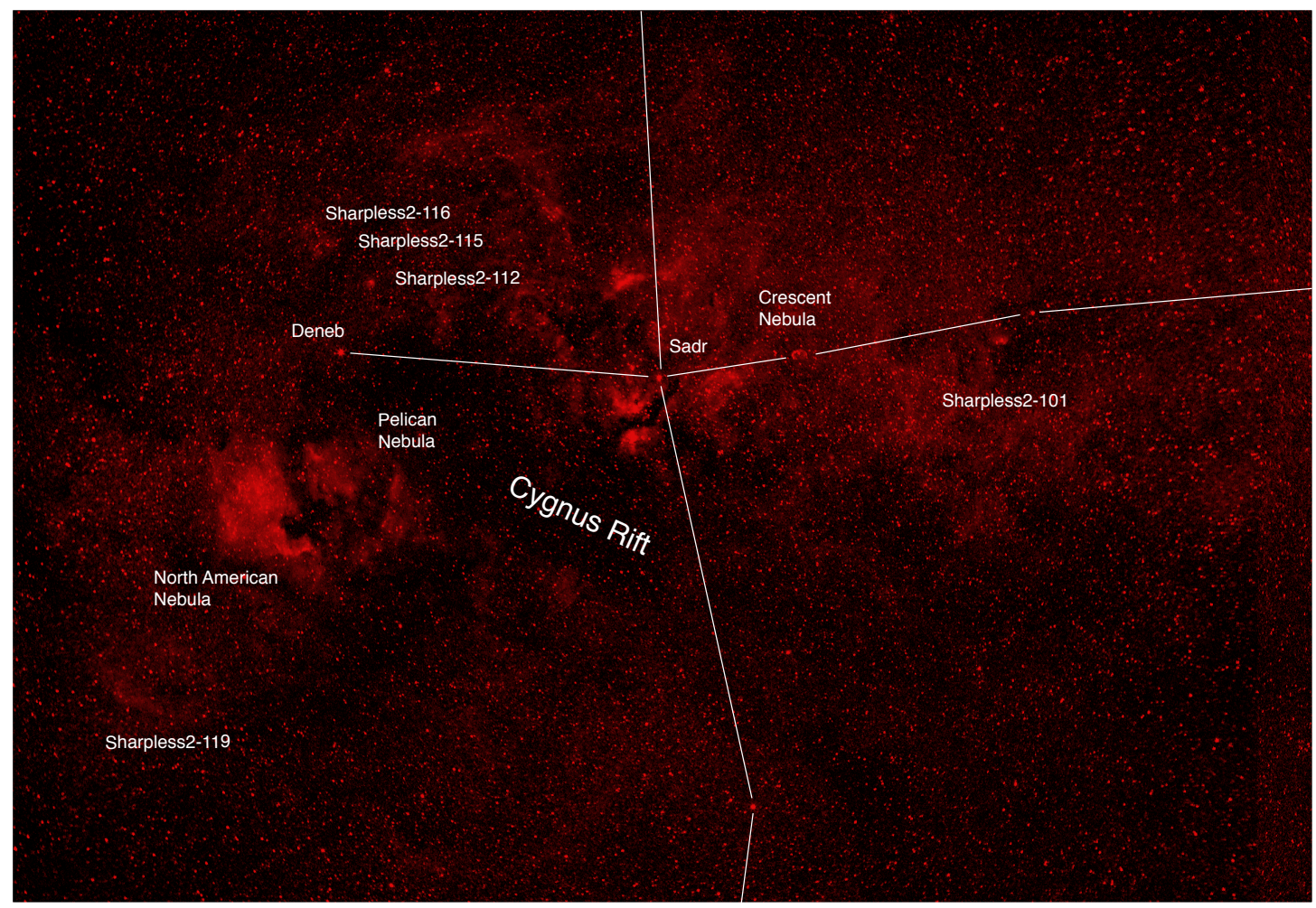

Figure 1. Unprocessed image of the Cygnus region in Hydrogen- $\alpha$. Some of the more notable objects in the region are labeled and the constellation Cygnus is outlined.

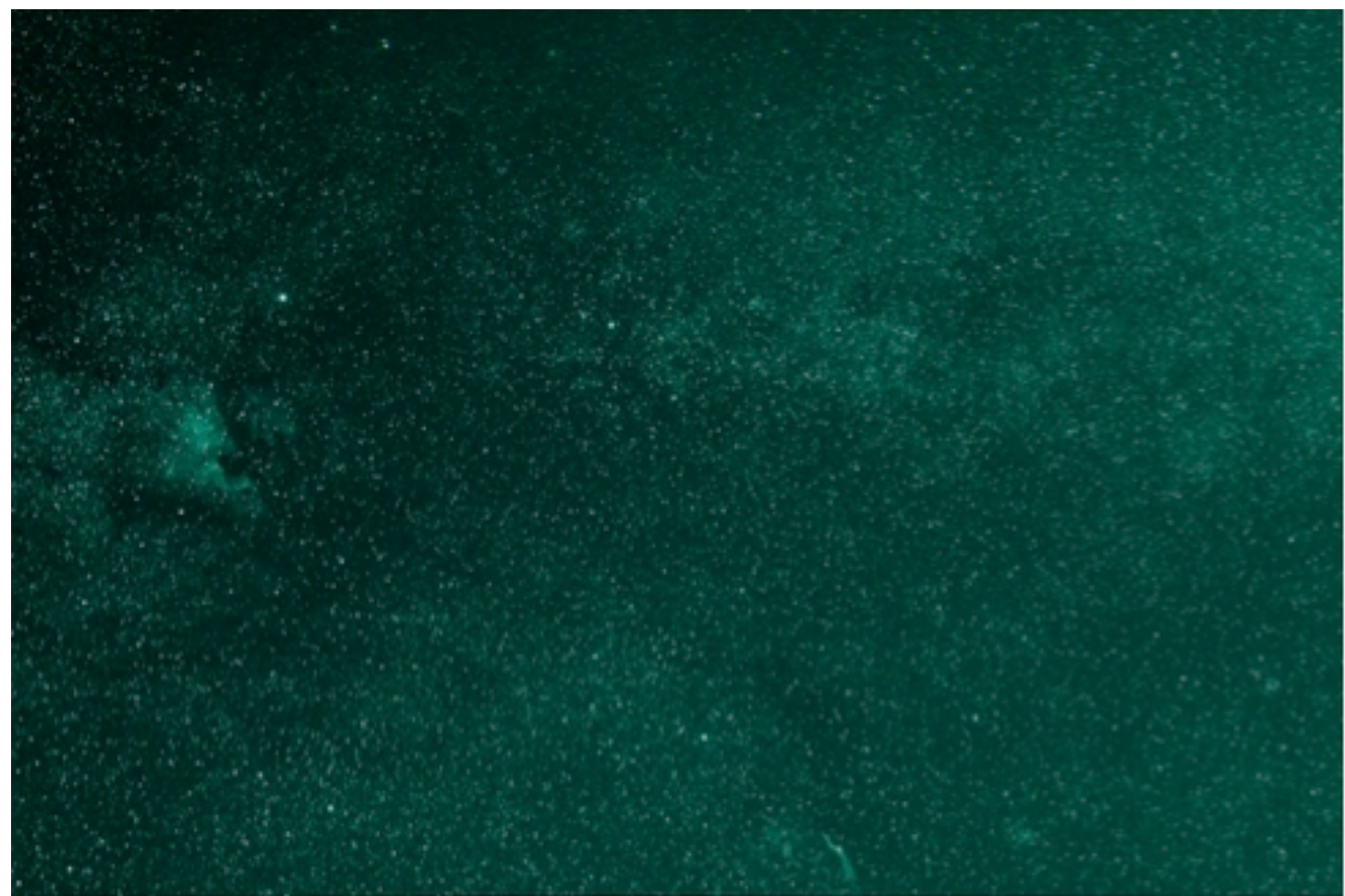

Figure 2. Oxygen III image of the Cygnus Superbubble. A part of the well-known supernova remnant, the Veil nebula, is shown at the bottom of this image. 
The conditions of the interstellar matter found in active star-formation sites can be analyzed based on the intensity of specific emission lines. For example, photometry in the Balmer emission lines, similar to the work done for some galactic and extragalactic nebulosities by Caplan and Deharveng (1985) and Cox, Deharveng and Caplan (1987), can be used to study the dust associated with the star-forming regions. Similarly, the morphology of a star-forming field at Hydrogen- $\alpha$ (Ho hereafter) and doubly ionized oxygen [O III] helps to better understand the physical condition and evolutionary stage of the nebulae around very energetic stars (see for example Gruendl et al., 2000).

\section{Results}

We utilize wide-field astrophotography equipment to image the Cygnus superbubble in $\mathrm{H} \alpha$ (656 $\mathrm{nm})$, [OIII] $(500.7 \mathrm{~nm})$ and $\mathrm{H} \beta(486 \mathrm{~nm})$ emission lines using 12-nm FWHM (Full Width at Half Maximum) filters. The equipment used was a computer-controlled guided telescope mount in combination with a modified DSLR camera and $50 \mathrm{~mm}$ F1.4 camera lens (Fig. 3). This allowed for most of the Cygnus star-forming field to be captured. DSLR's are designed to only capture visible light even though the sensors are sensitive into ultraviolet and infrared wavelengths. The cameras are built with an internal ultraviolet and infrared (UV/IR) cut filter to accomplish this. To allow for the capturing of images outside of the visual band, the UV/IR cut filter was removed and replaced with a filter from Baader Planetarium, which allows $400 \mathrm{~nm}$ to $700 \mathrm{~nm}$ light through. The Baader filter allowed for $99 \%$ of $\mathrm{H \alpha}$ light to pass through versus $25 \%$ with the original filter. The observations were performed during summer and fall of 2014 from various sites in Wisconsin. Nearly 40 images and 100 calibration frames were obtained to produce the combined science images.

Fig. 4 shows the ratio of $\mathrm{H} \alpha$ to $\mathrm{H} \beta$ emission, which traces the overall distribution of the interstellar dust. Both $\mathrm{H} \alpha$ and $\mathrm{H} \beta$ are recombination lines providing the overall distribution of

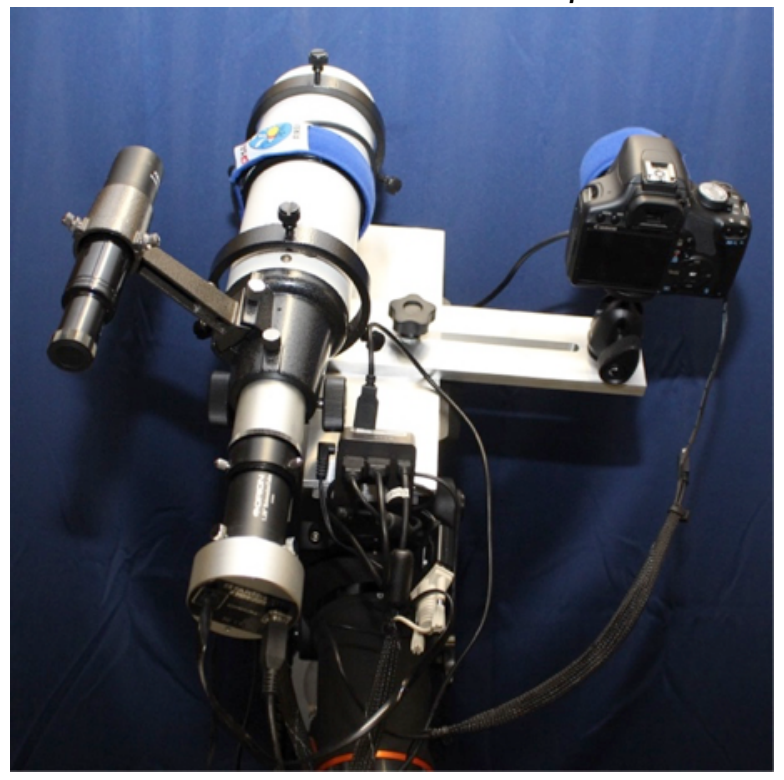

Figure 3. Research telescope. The guide-scope and camera on the left ensure accurate tracking while imaging. On the right is the modified DSLR camera used to collect the data. ionized material. The interstellar extinction selectively changes the intensities of the observed spectral lines and can be calculated comparing the observed Balmer decrement to the theoretical one. Fig. 4 is constructed in a way that regions of high extinction appear dark. One can trace the high-extinction filaments across the field. This comparison between the $\mathrm{H} \alpha$ and $\mathrm{H} \beta$ emission indicates that the largest extinction in the field is found in the North America Nebula and around the young cluster NGC 6910.

The [OIII] line at $500.7 \mathrm{~nm}$ is driven by collisional excitation and its intensity increases with the temperature of the interstellar gas. An [OIII] image of the Cygnus field is shown in Fig. 2, where part of a well-known supernova remnant, the Veil Nebula, is seen at the bottom. A comparison between the $\mathrm{H} \alpha$ and [OIII] 
emission would trace regions where the physical conditions change rapidly due to supernova shock fronts and strong stellar radiation. The preliminary processing of these images suggests no measurable offset between the $\mathrm{H} \alpha$ and [OIII] emission peaks, which probably implies shock fronts propagating into dense medium. The most intense [OIII] emission we detected was in the North America Nebula, which possibly indicates a presence of low-density high-temperature gas surrounded by a denser, cooler gas.

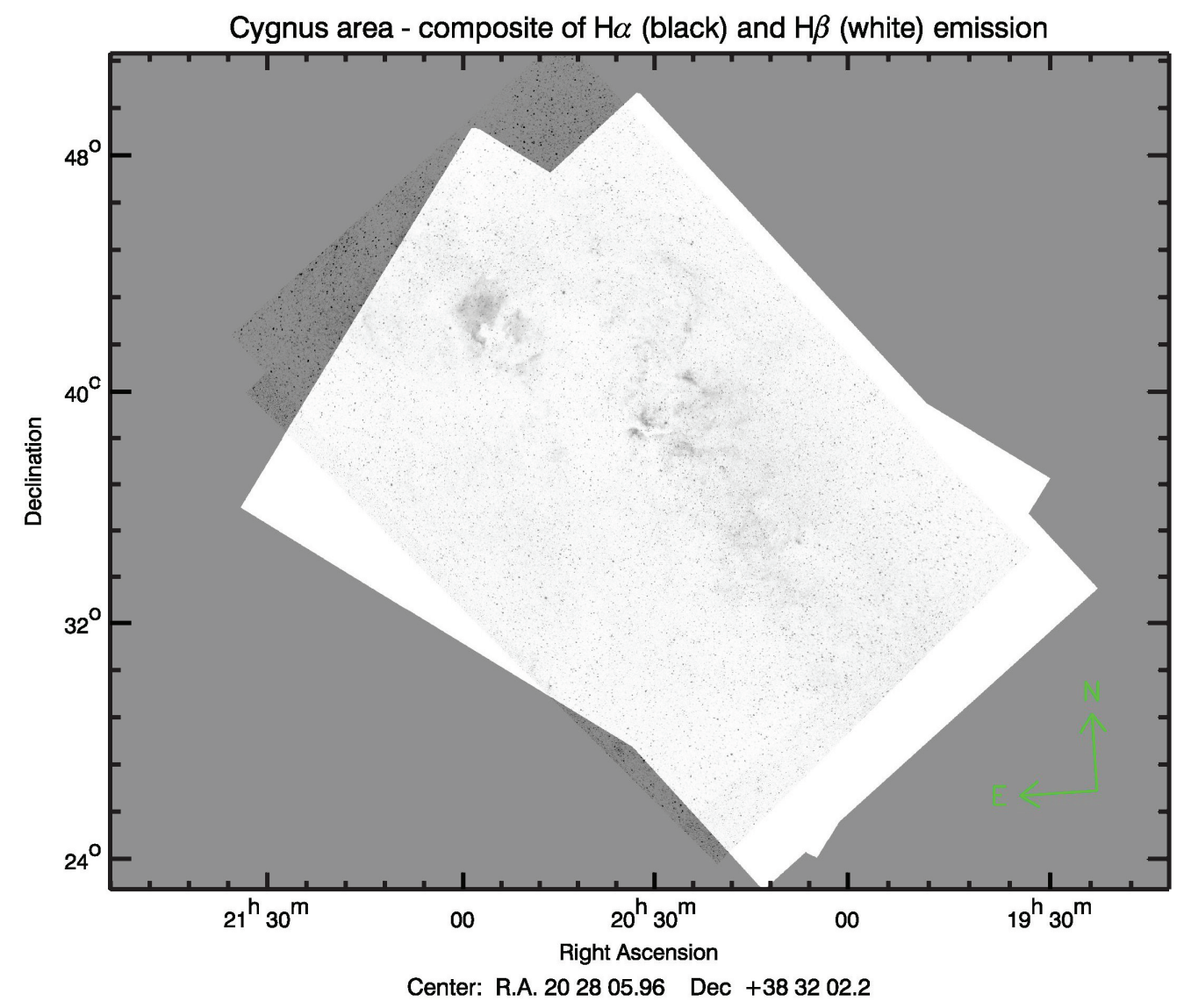

Figure 4. Ratio of the intensity of the $\mathrm{H} \alpha$ emission to the $\mathrm{H} \beta$ emission. Darker regions are where more extinction is present.

\section{Future work}

Depending on ambient conditions, a cool-down time of between five and ten minutes is needed to keep the image sensor at a consistent temperature and simplify noise removal. Work is currently being done to improve the image quality and increase the speed with which they can be taken. A thermoelectric cooler is being designed and built to keep the image sensor at a consistent temperature and reduce the cool-down time between images. With the improved equipment, the data collection will continue including more star-forming fields. 
Acknowledgements: We are thankful to Dr. V. Golev for his help with data reduction that was instrumental for this project. UW Oshkosh small student-faculty collaborative grant is also acknowledged.

\section{References}

Caplan, J., Deharveng, L., 1985. "Absolute H-alpha and H-beta photometry of LMC HII regions." Astronomy \& Astrophysics Supplement Series, 62, 63. http://adsabs.harvard.edu/abs/1985A\&AS...62...63C

Cox, P., Deharveng, L., Caplan, J. 1987. "Extinction and reddening towards compact Galactic HII regions." Astronomy \& Astrophysics, $171, \quad 277$. http://adsabs.harvard.edu/abs/1987A\&A...171..277C

Gruendl, R. A., Chu, Y. H., Dunne, B. C., \& Points, S. D. 2000. “A morphological diagnostic for dynamical evolution of Wolf-Rayet bubbles." The Astronomical Journal, 120(5), 2670. http://dx.doi.org/10.1086/316830

Sharpless, S. 1959. “A Catalogue of H II Regions.” Astrophysical Journal Supplement, 4, 257. http://dx.doi.org/10.1086/190049

Uyaniker, B., Fürst, E., Reich, W., Aschenbach, B., Wielebinski, R., 2001, "The Cygnus superbubble revisited." Astronomy \& Astrophysics, $371,675$. http://dx.doi.org/10.1051/0004-6361:20010387 